\title{
Controversies in the Locoregional Management of Head and Neck Cancer
}

\author{
David M. Brizel, MDa; William Lydiatt, MD ; and A. Dimitrios Colevas, MD; Durham, North Carolina; \\ Omaha, Nebraska; and Stanford, California
}

\author{
Key Words \\ Head and neck cancer, oropharynx, chemoradiation, neck \\ dissection, PET scan, cisplatin, cetuximab
}

\begin{abstract}
Head and neck cancer (HNC) is a heterogeneous combination of various sites and types of disease. This manuscript elaborates on $3 \mathrm{im}-$ portant and current issues: the emerging role of human papilloma virus (HPV) in oropharyngeal cancer (OPC), current considerations in systemic therapy for advanced disease, and evolving treatment of the neck. Exogenous carcinogens, most notably tobacco, have classically been implicated in the development of HNC. A large increase in the incidence of OPC has occurred in the past few decades, predominantly in nontobacco users, and is caused by HPV. This disease is unique in many respects and presents an opportunity for novel therapeutic approaches. Because the prognosis for HPV-related HNC is better, regardless of whether surgery or radiation is used as the primary therapy, the reduction of treatment-related morbidity has assumed increasing importance and provides unique opportunities and challenges for de-escalation of therapies. Radiotherapy (RT) and concurrent cisplatin is the most commonly used nonsurgical platform for locally advanced disease. New data suggest that viable alternatives exist to the typical 3 cycles of bolus high-dose cisplatin. The role of RT and concurrent taxanes remains less understood. Similarly, the value of integrating epidermal growth factor inhibition and concurrent chemoradiation is under continuing investigation. The use of PET scanning is changing the traditional use of adjuvant neck dissection after RT or chemoradiation. Recent data support the use of surgery in the presence of a positive posttreatment PET, and observation in the setting of a negative posttreatment scan. (JNCCN 2011;9:653-662)
\end{abstract}

From the apepartment of Radiation Oncology, Duke Cancer Institute, Duke University Medical Center, Durham, North Carolina; bUNMC Eppley Cancer Center at The Nebraska Medical Center, Omaha, Nebraska; and 'Stanford Comprehensive Cancer Center, Stanford, California.

Submitted January 24, 2011; accepted for publication February 28, 2011.

The authors have disclosed that they have no financial interests, arrangements, or affiliations with the manufacturers of any products discussed in this article or their competitors.

Correspondence: David M. Brizel, MD, Duke Cancer Institute, Department of Radiation Oncology; Box 3085, Duke University Medical Center, Durham, NC 27710. E-mail: david.brizel@duke.edu

\section{Oropharynx Cancer: New Challenges and New Opportunities}

Head and neck cancer (HNC) comprises malignancies of the oral cavity, paranasal sinuses, salivary glands, oropharynx, nasopharynx, larynx, and hypopharynx. The predominant histology of mucosal-based $\mathrm{HNC}$ is squamous cell carcinoma. Worldwide, more than 600,000 cases occur annually. Classically, $\mathrm{HNC}$ has been a disease of carcinogen-induced genetic alterations; specifically, mutations and deletions of oncogenes and tumor suppressor genes. The primary carcinogen is tobacco, either alone or in concert with other risk factors such as alcohol, betel or areca nut, industrial chemicals, heavy metals, and other carcinogens. These causative insults are often compounded by host factors such as poor nutrition, genetic susceptibility, and immunosuppression.

A historic exception has been Epstein-Barr virus (EBV)-associated nasopharyngeal carcinoma centered in Southeast Asia. This cancer is the most common malignancy in China and has a different natural history and response to treatment. Genetic and epigenetic factors combined with EBV infection result in a malignancy that has unique histologic and biologic properties. These differences are reflected in a separate staging system from other HNCs (AJCC $7^{\text {th }}$ Edition). In fact, the WHO classifies nasopharyngeal carcinoma into 3 types: WHO I nasopharyngeal carcinoma is the classic keratinizing squamous cell carcinoma similar to oral cavity cancers and has similar risk factors; WHO II is nonkeratinizing and is associated with EBV; and WHO III is undifferentiated carcinoma.

Over the past 3 to 4 decades, HNC caused by human papilloma virus (HPV) has emerged as a third unique entity. Declining rates of tobacco use in the West have resulted in a decreased incidence of malignancy in most head and neck anatomic subsites, but there has been an increase in the absolute and relative incidence of oro- 
Brizel et al.

pharyngeal cancer (OPC). Numerous investigators have shown an association between HPV and these cancers. HPV-related OPC presents in a fashion that is more typical of nasopharyngeal carcinoma than tobacco-associated cancer in both the oropharynx and other head and neck sites (see the article by Sturgis and Ang in this issue). Characteristics include an improved prognosis, lower rate of second primary tumors, younger age at diagnosis, less frequent smoking history, and fewer medical comorbidities.

The different demographics of this population make it likely that these patients will live for many years after successful definitive therapy, irrespective of the treatment modality used. Similar to earlystage Hodgkin's lymphoma, long-term side effects assume increasing prominence. ${ }^{1}$ Issues such as progressive dysphasia, cranial neuropathy, dysgeusia, xerostomia, progressive fibrosis in the pharynx or neck, dysphagia, depression, neurocognitive decline, ototoxicity, nephrotoxicity, and peripheral neuropathy must be evaluated acutely, chronically, and consistently.

The unique nature of HPV-related OPC suggests the need for a reassessment of existing treatment paradigms. The challenge is to develop new, less-intensive treatment programs that can reduce treatment-induced morbidity without compromising survival. Reduction in overall radiation dose, elimination of cisplatin-based concurrent systemic therapy, use of alternative systemic agents such as cetuximab, or an increased reliance on primary surgical therapy instead of (chemo)radiation have all been proposed. The crux of the debate is whether clinical equipoise exists with respect to various current and experimental treatment regimens.

Surgery has historically been the workhorse of HNC treatment. Over the past 20 years, the treatment of OPC has been largely supplanted by radiation-based therapy. It is well established that toxicity and control are directly related to radiation dose and the presence of accompanying systemic therapy. ${ }^{2,3}$ Thus, a reduction in either could lead to potentially improved functional outcomes but might also compromise anticancer effects. The advent of transoral robotic surgery (TORS) has renewed interest in the use of surgery for OPC. ${ }^{4}$ It is important to realize, however, that the specifics of the operative technique do not change the overall dynamic of whether the therapy is equivalent.
Transoral resection offers substantial advantages over transpharyngeal resections because it avoids disruption of sensory and muscular innervation of the pharyngeal mucosa and musculature. This results in substantially improved swallowing function and a lower incidence of perioperative complications. The traditional approach has been to use headlight illumination and direct visualization with oral and pharyngeal retraction. Access to the tumor can be difficult in certain patients with small mouths or large teeth, and when in locations such as deep base of tongue and extensive tonsil primaries. The use of endoscopic laser surgery and TORS have helped address the access challenge and possibly increase the number of patients for whom surgery would be an appropriate treatment option.., 5

The NCCN Clinical Practice Guidelines in Oncology (NCCN Guidelines) for Head and Neck Cancers (in this issue; to view the most recent version, visit the NCCN Web site at www.NCCN.org) recommend either primary radiation therapy or surgical therapy for early-stage OPC. As the $\mathrm{T}$ and/or $\mathrm{N}$ stage increases, the preferred modality is concurrent systemic therapy with radiation. ${ }^{6}$ Ongoing and future trials must address the increasingly common OPC, both in terms of overall and disease-specific survival and of equal importance with respect to long-term functional outcome. Conversely, HPV-negative, smoking-associated OPC must also be examined with respect to improved local and regional control and overall survival.

Equipoise ${ }^{7,8}$ must first exist with respect to survival for comparing surgical and nonsurgical strategies for HPV-positive cancers. The most striking feature of HPV-positive OPC is the significantly improved prognosis. Using SEER data and differentiation status as a surrogate for HPV, Mehta et al..$^{9}$ recently observed that the percentage of poorly differentiated tumors increased significantly from the mid-1970s to mid-2000s, whereas the well-differentiated tumors decreased in incidence. The overall survival of these patients also improved to $57 \%$ compared with $15 \%$ for the well-differentiated group. This indirectly suggests that HPV-mediated tumors, which are known to be poorly differentiated, are increasing in incidence and that survival is improved relative to the well-differentiated tumors, which are more often associated with tobacco.

Additionally, numerous prospective trials using combinations of radiation with or without combina- 
tions of systemic therapy have shown substantially improved survival. ${ }^{10-12}$ For example, Fakhry et al. ${ }^{13}$ reported improved survival and disease-specific survival for OPC in HPV-positive patients in a clinical trial investigating induction chemotherapy and concurrent radiation chemotherapy. The survival benefit does not seem to hold up outside of the oropharynx, however. ${ }^{14}$ Surgical trials have also shown improvements in survival. ${ }^{8}$ Haughey ${ }^{15}$ showed a nearly $60 \%$ reduced risk of death in HPV-positive OPC compared with historical controls using transoral laser microsurgery in an abstract from the 2009 American Head and Neck Society meeting. Thus, improved survival might simply reflect the biology of the disease itself rather than the choice of a particular therapeutic modality. These findings suggest that equipoise does exist with respect to surgical and nonsurgical primary therapy for HPV-positive oropharyngeal squamous cell carcinoma.

How should a trial be conducted to simultaneously address the issues of survival and long-term organ function and quality of life? One intrinsic problem encountered is the vast difference in opinion regarding surgical and nonsurgical therapies. Preconceived notions on the part of both clinicians and patients can make enrollment onto randomized trials particularly difficult. Reaching consensus among investigators regarding actual treatment regimens to assess constitutes another hurdle. The NCI's Head and Neck Steering Committee is taking an active role in addressing these challenges.

\section{Radiotherapy and Concurrent Systemic Therapy}

The most common anticancer agents used concurrently with radiation in the curative treatment of squamous cell carcinoma of the head and neck (SCCHN) include cisplatin, carboplatin, paclitaxel, docetaxel, cetuximab, and 5-fluorouracil. Less commonly used agents include hydroxyurea and bleomycin. Cisplatin and carboplatin are by far the most common chemotherapy agents used in combination with radiation therapy for the treatment of patients with locoregionally advanced SCCHN.

The use of cisplatin dates back to the early 1970s when it became apparent that cisplatin as a single agent and combined with other agents caused significant cytoreduction in SCCHN. The initial report of chemotherapy involving cisplatin plus bleomycin by Wittes et al. ${ }^{16}$ in 1975 was soon followed by reports of combination treatments of cisplatin and radiation often in conjunction with other drugs. For example, a report by Randolph et al. ${ }^{17}$ in 1978 described a major response rate of $71 \%$ to cisplatin and bleomycin preceding radiation, with more than half of the patients experiencing complete response for many months after the end of radiation treatment.

The concurrent administration of cisplatin and radiation was recognized as having a high level of toxicity as early as 1981, and therefore some investigators thought that concurrent administration of these agents was not feasible. ${ }^{18}$ However, several subsequent studies showed the feasibility of concurrent cisplatin and radiation in patients with $\mathrm{HNC}$ when accompanied by vigorous intravenous hydration, aggressive electrolyte management, and nausea prophylaxis. ${ }^{19}$

Subsequently, many multi-institutional trials conducted in Europe and the United States in the 1980s and 1990s confirmed that the coadministration of high-dose $\left(75-100 \mathrm{mg} / \mathrm{m}^{2}\right)$ cisplatin and radiation in patients with SCCHN was both achievable and beneficial in settings beyond the highly selected populations seen at referral centers..$^{20,21}$ This feasibility was not limited to conventional once-daily radiation, but was also seen with newer more-aggressive accelerated or hyperfractionated radiation dosing schemes. ${ }^{22}$

The late 1980s and early 1990s saw a plethora of publications on randomized controlled trials of radiation alone versus radiation plus chemotherapy. The outcome of these trials varied, but several metaanalyses showed a consistent theme of increased response rates, increased disease control, and improved survival in the trials incorporating concurrent cisplatinbased chemoradiation. ${ }^{23-26}$ In aggregate, trials of chemotherapy plus radiation versus radiation alone showed a $4 \%$ absolute survival advantage at 5 years. However, when the analysis was restricted to concurrent cisplatin-based chemotherapy with radiation, an $8 \%$ absolute survival advantage at 5 years was observed. This benefit corresponded to a $19 \%$ reduction in the risk of death. Notably, the meta-analysis data sets represent more than 16,000 patients treated on more than 80 randomized controlled trials with median follow-up in excess of 5 years. Because most of these trials involved standard daily radiation fractionation, whether cisplatin-based chemoradiation would 
Brizel et al.

provide similar benefits in patients treated with more intensive accelerated or hyperfractionated radiation treatment plans is uncertain. Several randomized controlled trials have shown that a survival advantage from the addition of concurrent cisplatin to radiation persists in this setting. The relatively limited number of trials and heterogeneous mix of treatments makes the magnitude of benefit less uncertain, however. ${ }^{27-29}$ A meta-analysis of 32 randomized controlled trials with 10,225 patients in aggregate confirmed the advantage of chemoradiation over radiation when using either hyperfractionated or accelerated radiation. ${ }^{30} \mathrm{In}$ this meta-analysis, 5-fluorouracil outperformed cisplatin in terms of median survival benefit.

These data confirm that concurrent cisplatin plus radiation can be considered a nonsurgical standard of care for patients with locoregionally advanced SCCHN. The best dosing regimen for cisplatin is not well established, and is somewhat controversial, however. This uncertainty probably reflects an incomplete understanding of the molecular interactions between cisplatin and DNA, the pharmacokinetics of cisplatin, and the mechanism of cisplatinmediated radiosensitization.

Numerous dosing regimens ranging from high doses of 75 to $100 \mathrm{mg} / \mathrm{m}^{2}$ every 21 days ( 3 cycles) during radiation, to weekly intermediate doses of 30 to $40 \mathrm{mg} / \mathrm{m}^{2}$, to low daily doses ${ }^{31}$ of $6 \mathrm{mg} / \mathrm{m}^{2}$ have been clinically successful, ${ }^{27,32,33}$ but the high-dose regimen has been used most extensively in cooperative group trials. Recently, RTOG 0129 compared standard fractionation RT with 3 cycles of concurrent cisplatin and accelerated fractionation RT and 2 cycles of cisplatin in 721 patients. ${ }^{34}$ Efficacy and toxicity of these regimens were equivalent. One-fourth of the patients on the standard fractionation arm only received 2 cycles of cisplatin. A subset analysis showed no difference in outcome between these patients and those who received all 3 cycles. Similarly, no difference has been observed in postoperative chemoradiation trials as a function of 2 versus 3 cycles of cisplatin. . $^{35,36}$ Some investigators suggest that the common theme among these different cisplatin dosing schemes is that a minimum cumulative dose of $200 \mathrm{mg} / \mathrm{m}^{2}$ is necessary for efficacy in a concurrent therapy setting. ${ }^{37}$

Whether carboplatin provides as much benefit as cisplatin in this setting is unclear. Evidence shows that carboplatin has less anticancer activity than cisplatin in SCCHN and multiple other cancers when used without radiation in the metastatic or recurrent disease setting. ${ }^{38}$ One randomized controlled trial in SCCHN that compared cisplatin versus carboplatin administered concurrently with radiation suggested the same, but is less conclusive. ${ }^{39}$ A difference in outcomes with one versus the other agent is difficult to discern, especially when the dosing regimens for each drug are different across studies, sample sizes are small, and anatomic subsites are variable..$^{40-42}$ The most widely cited meta-analyses on chemoradiation in SCCHN do not adequately examine the difference between cisplatin and carboplatin, but at least one analysis strongly favors cisplatin in this setting. ${ }^{30}$

The use of radiation with concurrent singleagent carboplatin is encountered most commonly in the setting of sequential chemoradiation (induction cisplatin-based chemotherapy followed by radiotherapy and concurrent chemotherapy). Toxicity considerations usually preclude delivery of additional concurrent cisplatin with this treatment strategy. ${ }^{43}$ The roles of induction chemotherapy and sequential chemoradiation are both controversial and beyond the scope of this article.

Cetuximab, a chimeric monoclonal antibody $(\mathrm{MoAb})$ to the epidermal growth factor receptor (EGFR), has the distinction of being the only anticancer agent that is FDA approved specifically for use concurrently with radiation. A single randomized controlled trial of 424 patients in the curative setting combined with radiation, and one trial in the palliative setting in which this antibody was combined with cisplatin-based chemotherapy, have shown statistically significant and clinically meaningful improvements in progression-free and overall survival, respectively, from cetuximab. ${ }^{44-47}$ For patients with locoregional SCCHN, 5-year median overall survival was $9 \%$ higher in the cetuximab plus radiation arm versus the radiation alone arm. This benefit seems to be approximately the same magnitude as the survival benefit associated with concurrent cisplatin in the meta-analysis of cisplatin-based chemoradiation trials discussed earlier, and therefore some experts have proposed the hypothesis that cetuximab might be a less-toxic equiefficacious substitute for cisplatin in this setting. ${ }^{45}$

Subset analysis of the cetuximab plus radiation randomized controlled trial ${ }^{45,46}$ suggested that the benefit of cetuximab was most appreciable in patients with SCC of the oropharynx, raising the possi- 
Controversies in Locoregional Management

bility that HPV-associated cancers in particular may benefit from a combination treatment such as this. However, because this was a subset analysis and the specific HPV status of each patient's tumor was not known, this must be viewed as hypothesis-generating data rather than definitive evidence of specific benefits for HPV-positive patients.

Whether the populations of patients with SCCHN who benefit most from cisplatin are the same or different from the patients who would benefit most from cetuximab is currently unknown. Extrapolation of the clinical benefits of cetuximab to EGFR inhibitors as a class is premature. Data presented at the 2010 annual meeting of the European Society of Medical Oncology suggested that panitumumab, a monoclonal antibody of the IgG2 class directed against EGFR, did not show the same benefit as cetuximab when combined with platinum-based chemotherapy (Jan Vermorken, MD, PhD, personal communication, 2010). The addition of erlotinib and gefitinib, small molecule inhibitors of the tyrosine kinase domain of EGFR, to radiation or chemoradiation with both sequential and concurrent treatment regimens has been shown to be feasible, but the clinical benefit is unknown. ${ }^{48-50}$

Additional clinically relevant data concerning the role of anti-EGFR MoAbs in place of or combined with cisplatin and concurrent chemoradiation will likely be available in the next few years. Several pivotal phase III studies exploring various combinations of anti-EGFR MoAbs are approaching maturity. These include the Danish Head and Neck Cancer Group's study of radiation versus radiation plus zalutumumab in patients with stage I and II disease, and cisplatin-based chemoradiation $+/-$ zalutumumab in patients with stage III and IV disease. A randomized controlled trial of panitumumab versus cisplatin in combination with radiation is underway within the National Cancer Institute of Canada cooperative group. ${ }^{51}$ The US Radiation Therapy Oncology Group (RTOG) recently completed accrual to RTOG 0522,52 a randomized controlled trial of cisplatin alone versus cisplatin plus cetuximab in combination with radiation in patients with locoregionally advanced SCCHN. Because of the changing demographics of OPC in the West, these trials will almost surely be enriched with patients who have HPV-related disease.
Taxanes are among the most active drugs known against SCCHN, and both have been studied extensively preclinically as radiation sensitizers. ${ }^{53-57}$ They have also been studied in phase II trials in combination with radiation in patients with locoregional SCCHN, and combined with other agents, including cisplatin and cetuximab. . $^{5,58-63}$ These studies have, in aggregate, shown the feasibility of this approach and reported response rates and short-term survival data similar to those reported for cisplatin-based chemoradiation. Results presented at the ASTRO 2009 annual meeting ${ }^{64}$ from the RTOG 0234 trial, which compared cetuximab, cisplatin, and radiation with cetuximab, docetaxel, and radiation in the postsurgical setting, showed statistically indistinguishable progression-free and overall survival curves for these regimens, although the curves for the taxane-containing regimen were numerically superior to those for the cisplatin-containing regimens.

Another small randomized controlled trial of lowdose weekly paclitaxel plus radiation versus weekly cisplatin plus radiation in patients with locally advanced SCCHN showed no statistically significant difference in either complete response rate or short-term relapsefree survival between the arms. ${ }^{59}$ Unfortunately, despite more than a decade of awareness of the feasibility of concurrent taxane plus radiation treatment, and promising phase II data of regimens incorporating concurrent taxane and radiation treatment, no pivotal trials are planned that will address the role of the taxanes versus platinum or anti-EGFR MoAbs as potentially curative chemoradiation treatment.

In summary, the preponderance of clinical trial data supports the use of cisplatin as the favored agent when using concurrent chemoradiation for patients with locoregional SCCHN. The ideal dosing of cisplatin in this setting has not been established, but high-dose intermittent and more frequently administered lower dosing regimens have shown benefit. Although carboplatin, anti-EGFR MoAbs, and taxanes all have substantial preclinical and clinical data supporting their use in combination with radiation in patients with locoregionally advanced SCCHN, the absence of direct comparisons to cisplatin-based regimens prevents a definitive rank ordering of benefit of these alternatives, but carboplatin and cetuximab data are robust enough to warrant their designations as acceptable alternative standards in patients who are not candidates for cisplatin treatment. 
Brizel et al.

\section{Management of the Neck}

Management of cancer in the lymph nodes is complex. Confusion often arises largely from the failure to recognize whether the proposed intervention, be it surgery or radiation, is part of the definitive or the adjuvant component of therapy. Surgery and radiotherapy as single modalities are equivalent for control of early-stage neck disease (N0-N1) under most conditions. Consequently, the modality chosen for the initial treatment of the neck should be the same one used for the management of the primary tumor site. The value of this strategy is that it creates a circumstance in which the patient may only require single-modality therapy. Which nodal stations are resected or irradiated should be based on the primary tumor anatomic site, initial stage, and risk or presence of nodal involvement.

Single-modality is preferable to multimodality therapy, when clinically appropriate, especially for early-stage disease, because the morbidity of treatment increases in conjunction with the use of multiple therapeutic modalities. Illustrative scenarios in which surgery alone constitutes appropriate therapy for the neck include negative margins/no perineural invasion at the primary site and either a pathologic NO neck or pathologic N1 neck with no extracapsular extension. Definitive chemoradiation would usually suffice as adequate treatment for the neck in the context of a complete response to chemoradiation at the primary site for NO presentations and in the setting of a complete response at the primary and neck for $\mathrm{N}+$ disease. The corollary is that adjuvant postoperative irradiation would be indicated in circumstances, such as in the presence of multiple positive lymph nodes without extracapsular extension, whereas adjuvant chemoradiation would be indicated in the presence of one or more lymph nodes with extracapsular extension.

Historically, combinations of surgery and radiotherapy were superior to either modality alone for controlling N2 and N3 disease, ${ }^{65}$ mainly because unfavorable histopathology or residual disease were more likely to occur in the setting of these more advanced nodal presentations. More recently, both the RTOG and EORTC prospectively showed that adjuvant postoperative concurrent chemoradiation is more efficacious than adjuvant radiation alone when extracapsular nodal extension is present. . $^{35,36}$
Adjuvant neck dissection should be performed when residual nodal disease persists after definitive chemoradiation, but the decision-making can nonetheless be controversial. ${ }^{66-71}$ Issues that must be addressed in making the determination to operate include the method of assessing whether a complete response has occurred in the neck, the ideal time to make this evaluation, and the type of neck dissection to perform. . $^{66,67}$

The evaluation of the neck has evolved extensively over several decades. Classically, physical examination was the primary assessment technique. A clinical complete response is highly predictive of long-term regional disease control for N1 disease. Clinical response is considerably less valuable in confirming the presence or absence of residual disease after treatment of $\mathrm{N} 2$ and N3 presentations, in which factors such as treatment-induced inflammation and edema may confound the interpretation of a residual neck mass. False-positive (residual mass palpated, pathologically negative at surgery) and false-negative (no residual mass palpated, pathologically positive at surgery) rates in this setting are 25\% to 30\%. Many groups advocated adjuvant neck dissection postchemoradiation because of the inaccuracy of clinical assessment, and some showed survival benefits associated with the performance of neck dissection even in the presence of a clinical complete response. ${ }^{67}$

CT and PET scanning have markedly improved the ability to evaluate neck response post-chemoradiation. The probability that residual disease will be identified in a pathologic specimen is 3\% to $5 \%$ if a PET or contrast-enhanced CT is negative, and the likelihood of a neck recurrence in a nondissected neck is similarly small in the context of a negative post-chemoradiation scan. ${ }^{72-74}$

The elapsed time from the completion of chemoradiation to the performance of the scan is an important consideration. Some surgeons advocate for the performance of adjuvant neck surgery within an 8 - to 12-week window after the completion of chemoradiation, with radiographic imaging thus performed 6 to 8 weeks post-chemoradiation. The rationale for this philosophy is that beyond this time point, treatmentinduced scarring and fibrosis make the surgery more difficult and increase its morbidity. Evidence supporting this window concept, which evolved during the era in which large-volume irradiation and radical neck dissection were de rigueur, is sparse. Improve- 
ments in radiotherapeutic (intensity-modulated irradiation) and surgical techniques (modified and selective neck dissection), both of which reduce the volume of normal noncancerous tissue that is treated or removed, have rendered it largely obsolete.

The interval from the completion of chemoradiation to the performance of radiographic imaging is also important because a positive scan will be used to select patients who will undergo neck surgery. The false-positive rate of a PET scan that is obtained 6 to 8 weeks after treatment completion approaches $50 \%$, but falls to only $10 \%$ to $15 \%$ if the imaging is performed 12 weeks posttreatment, thus reducing the number of surgeries on patients with no pathologic residual disease. ${ }^{73,74}$ This reduced false-positive rate when scans are delayed an additional 4 weeks is probably caused by the resolution of chemoradiation-induced inflammation, which can cause increased uptake on a PET. A recent Australian study of 112 node-positive patients prospectively evaluated the role of FDG-PET performed 12 weeks after completion of chemoradiation in the assessment and management of the neck. N stage was N2 in 84 (75\%) patients and N3 in 14 (12\%). All 62 (55\%) of the patients who experienced a clinical and radiographic complete response were followed up, and none experienced a recurrence; 50 (45\%) had residual abnormalities on CT scan with a median size of $15 \mathrm{~mm}$. PET was negative for 41 of these patients, who were subsequently observed with no nodal failures seen. Neck dissection was performed in 8 of 9 patients with residual abnormalities on PET, with viable disease identified in $6 .{ }^{75}$

Adjuvant neck dissection improves regional control and survival in patients who have residual disease after completing primary nonsurgical therapy. ${ }^{67,70,76}$ The benefit may be especially pronounced in patients who initially present with $\mathrm{N} 3$ disease. ${ }^{66}$ Pathologic confirmation of complete response at the primary site should be obtained before adjuvant neck dissection is performed. The presence of persistent primary site disease would require a more comprehensive surgical salvage procedure for both the primary and neck. The most common approach is to assess the primary through biopsy with frozen section interpretation. If the specimen is negative, then the surgeon should proceed to neck dissection.

Historically, radical neck dissection was used, which removes the level I through $\mathrm{V}$ lymph nodes along with the sternocleidomastoid, jugular vein, and spinal accessory nerve. This procedure was commonly indicated in the primary surgical management of node-positive presentations, particularly N2-N3 disease, in which en bloc removal of this anatomy was necessary to ensure adequate removal of all gross disease. Modified neck dissections typically spare one or more of the anatomic structures that are resected in a radical neck dissection, but still remove the same nodal groups. Selective neck dissection further spares one or more of the lymph node groups that are removed in the radical procedure. ${ }^{77}$ Modified and selective neck dissections now constitute the predominant forms of adjuvant therapy for residual neck disease. The rationale for performing less-intensive surgery in the adjuvant setting is that chemoradiation is a very effective treatment for subclinical deposits of disease, and therefore obviates the need for surgical clearance of nodal levels that did not harbor gross disease on initial presentation. Moreover, the less-aggressive surgery reduces the risk of long-term morbidity given the intensive radiation, with or without chemotherapy, that has already been delivered. Efficacy is not compromised with the use of modified or selective procedures, as opposed to with radical neck dissection..$^{70,71,78}$

\section{Conclusions}

HNC represents a wide spectrum of diseases with multiple origins. The 3 evolving areas discussed represent some of the most significant challenges in head and neck oncology today. The evolution of different treatment strategies reflects the need for comprehensive management of patients, with specific attention to optimizing both the chance of cure and maintenance of organ function. Although progress is dramatic, new questions are arising that necessitate comprehensive strategies in research and treatment; this is the challenge to today's radiation, surgical, and medical oncologists.

\section{References}

1. Armitage JO. Early-stage Hodgkin's lymphoma. N Engl J Med 2010;363:653-662.

2. Bentzen SM, Rosenthal DI, Weymuller EA, Trotti A. Increasing toxicity in nonoperative head and neck cancer treatment: investigations and interventions. Int J Radiat Oncol Biol Phys 2007;69:S79-82. 
Brizel et al.

3. Bentzen SM, Trotti A. Evaluation of early and late toxicities in chemoradiation trials. J Clin Oncol 2007;25:4096-4103.

4. O'Malley BW Jr, Weinstein GS, Snyder W, Hockstein NG. Transoral robotic surgery (TORS) for base of tongue neoplasms. Laryngoscope 2006;116:1465-1472.

5. Cohen MA, Weinstein GS, O'Malley BW Jr, et al. Transoral robotic surgery and human papillomavirus status: oncologic results. Head Neck 2011;33:573-580.

6. Pfister DG, Ang KK, Brixel D, et al. NCCN Clinical Practice Guidelines in Oncology: Head and Neck Cancers. Version 2, 2011. Available at: http://www.nccn.org/professionals/physician_gls/pdf/ head-and-neck.pdf. Accessed March 31, 2011.

7. Djulbegovic B. The paradox of equipoise: the principle that drives and limits therapeutic discoveries in clinical research. Cancer Control 2009; 16:342-347.

8. Djulbegovic B, Cantor A, Clarke M. The importance of preservation of the ethical principle of equipoise in the design of clinical trials: relative impact of the methodological quality domains on the treatment effect in randomized controlled trials. Account Res 2003;10:301-315.

9. Mehta V, Yu GP, Schantz SP. Population-based analysis of oral and oropharyngeal carcinoma: changing trends of histopathologic differentiation, survival and patient demographics. Laryngoscope 2010;120:2203-2212.

10. Hong AM, Dobbins TA, Lee CS, et al. Human papillomavirus predicts outcome in oropharyngeal cancer in patients treated primarily with surgery or radiation therapy. $\mathrm{Br} \mathrm{J}$ Cancer 2010;103:1510-1517.

11. Ang KK, Harris J, Wheeler R, et al. Human papillomavirus and survival of patients with oropharyngeal cancer. N Engl J Med 2010;363:24-35.

12. Attner P, Du J, Nasman A, et al. Human papillomavirus and survival in patients with base of tongue cancer. Int J Cancer, in press.

13. Fakhry C, Westra WH, Li S, et al. Improved survival of patients with human papillomavirus-positive head and neck squamous cell carcinoma in a prospective clinical trial. J Natl Cancer Inst 2008;100:261-269.

14. Allen CT, Lewis JS Jr, El-Mofty SK, et al. Human papillomavirus and oropharynx cancer: biology, detection and clinical implications. Laryngoscope 2010;120:1756-1772.

15. Haughey B. Transoral laser microsurgery as primary treatment of advanced stage oropharyngeal cancer: a United States Multicenter Study [abstract]. Presented at the American Head and Neck Society Meeting; May 27-31, 2009; Phoenix, Arizona. Abstract S032.

16. Wittes RE, Brescia F, Young CW, et al. Combination chemotherapy with cis-diamminedichloroplatinum (II) and bleomycin in tumors of the head and neck. Oncology 1975;32:202-207.

17. Randolph VL, Vallejo A, Spiro RH, et al. Combination therapy of advanced head and neck cancer: induction of remissions with diamminedichloroplatinum (II), bleomycin and radiation therapy. Cancer 1978;41:460-467.

18. Creagan ET, Fountain KS, Frytak S, et al. Concomitant radiation therapy and cis-diamminedichloroplatinum (II) in patients with advanced head and neck cancer. Med Pediatr Oncol 1981;9:119120.

19. Keizer HJ, Karim AB, Njo $\mathrm{KH}$, et al. Feasibility study on daily administration of cis-diamminedichloroplatinum(II) in combination with radiotherapy. Radiother Oncol 1984;1:227-234.
20. Adelstein DJ, Kalish LA, Adams GL, et al. Concurrent radiation therapy and chemotherapy for locally unresectable squamous cell head and neck cancer: an Eastern Cooperative Oncology Group pilot study. J Clin Oncol 1993;11:2136-2142.

21. Adelstein DJ, Sharan VM, Damm C, et al. Concurrent radiation therapy, 5-fluorouracil, and cisplatin for stage II, III, and IV, nodenegative, squamous cell head and neck cancer. Results and surgical implications. Cancer 1992;70:2685-2690.

22. Brizel DM, Leopold KA, Fisher SR, et al. A phase I/II trial of twice daily irradiation and concurrent chemotherapy for locally advanced squamous cell carcinoma of the head and neck. Int J Radiat Oncol Biol Phys 1994;28:213-220.

23. El-Sayed S, Nelson N. Adjuvant and adjunctive chemotherapy in the management of squamous cell carcinoma of the head and neck region. A meta-analysis of prospective and randomized trials. J Clin Oncol 1996;14:838-847.

24. Pignon JP, Bourhis J, Domenge C, Designe L. Chemotherapy added to locoregional treatment for head and neck squamous-cell carcinoma: three meta-analyses of updated individual data. $\mathrm{MACH}$ NC Collaborative Group. Meta-Analysis of Chemotherapy on Head and Neck Cancer. Lancet 2000;355:949-955.

25. Pignon JP, le Maitre A, Bourhis J. Meta-analyses of chemotherapy in head and neck cancer (MACH-NC): an update. Int J Radiat Oncol Biol Phys 2007;69:S112-114.

26. Pignon JP, le Maitre A, Maillard E, Bourhis J. Meta-analysis of chemotherapy in head and neck cancer (MACH-NC): an update on 93 randomised trials and 17,346 patients. Radiother Oncol 2009;92:4-14.

27. Brizel DM, Albers ME, Fisher SR, et al. Hyperfractionated irradiation with or without concurrent chemotherapy for locally advanced head and neck cancer. N Engl J Med 1998;338:17981804.

28. Michiels S, Le Maitre A, Buyse M, et al. Surrogate endpoints for overall survival in locally advanced head and neck cancer: metaanalyses of individual patient data. Lancet Oncol 2009;10:341350.

29. Staar S, Rudat V, Stuetzer H, et al. Intensified hyperfractionated accelerated radiotherapy limits the additional benefit of simultaneous chemotherapy—results of a multicentric randomized German trial in advanced head-and-neck cancer. Int J Radiat Oncol Biol Phys 2001;50:1161-1171.

30. Budach W, Hehr $\mathrm{T}$, Budach $\mathrm{V}$, et al. A meta-analysis of hyperfractionated and accelerated radiotherapy and combined chemotherapy and radiotherapy regimens in unresected locally advanced squamous cell carcinoma of the head and neck. BMC Cancer 2006;6:28.

31. Jeremic B, Shibamoto Y, Milicic B, et al. Hyperfractionated radiation therapy with or without concurrent low-dose daily cisplatin in locally advanced squamous cell carcinoma of the head and neck: a prospective randomized trial. J Clin Oncol 2000;18:1458-1464.

32. Watkins JM, Zauls AJ, Wahlquist AH, et al. Low-dose weekly platinum-based chemoradiation for advanced head and neck cancer. Laryngoscope 2010;120:236-242.

33. Huguenin P, Beer KT, Allal A, et al. Concomitant cisplatin significantly improves locoregional control in advanced head and neck cancers treated with hyperfractionated radiotherapy. J Clin Oncol 2004;22:4665-4673.

34. Gan HK, Bernstein LJ, Brown J, et al. Cognitive functioning after radiotherapy or chemoradiotherapy for head-and-neck cancer. Int J Radiat Oncol Biol Phys, in press. 
Controversies in Locoregional Management

35. Bernier J, Domenge C, Ozsahin M, et al. Postoperative irradiation with or without concomitant chemotherapy for locally advanced head and neck cancer. N Engl J Med 2004;350:1945-1952.

36. Cooper JS, Pajak TF, Forastiere AA, et al. Postoperative concurrent radiotherapy and chemotherapy for high-risk squamous-cell carcinoma of the head and neck. N Engl J Med 2004;350:19371944.

37. Ang KK. Concurrent radiation chemotherapy for locally advanced head and neck carcinoma: are we addressing burning subjects? J Clin Oncol 2004;22:4657-4659.

38. De Andres L, Brunet J, Lopez-Pousa A, et al. Randomized trial of neoadjuvant cisplatin and fluorouracil versus carboplatin and fluorouracil in patients with stage IV-MO head and neck cancer. J Clin Oncol 1995;13:1493-1500.

39. Fountzilas G, Ciuleanu E, Dafni U, et al. Concomitant radiochemotherapy vs radiotherapy alone in patients with head and neck cancer: a Hellenic Cooperative Oncology Group phase III study. Med Oncol 2004;21:95-107.

40. Chitapanarux I, Lorvidhaya V, Kamnerdsupaphon $P$, et al. Chemoradiation comparing cisplatin versus carboplatin in locally advanced nasopharyngeal cancer: randomised, non-inferiority, open trial. Eur J Cancer 2007;43:1399-1406.

41. Homma A, Shirato H, Furuta Y, et al. Randomized phase II trial of concomitant chemoradiotherapy using weekly carboplatin or daily low-dose cisplatin for squamous cell carcinoma of the head and neck. Cancer J 2004;10:326-332.

42. Jeremic B, Shibamoto Y, Stanisavljevic B, et al. Radiation therapy alone or with concurrent low-dose daily either cisplatin or carboplatin in locally advanced unresectable squamous cell carcinoma of the head and neck: a prospective randomized trial. Radiother Oncol 1997;43:29-37.

43. Posner MR, Hershock DM, Blajman CR, et al. Cisplatin and fluorouracil alone or with docetaxel in head and neck cancer. $\mathrm{N}$ Engl J Med 2007;357:1705-1715.

44. Bonner JA, Harari PM, Giralt J, et al. Radiotherapy plus cetuximab for squamous-cell carcinoma of the head and neck. N Engl J Med 2006;354:567-578.

45. Bonner JA, Harari PM, Giralt J, et al. Radiotherapy plus cetuximab for locoregionally advanced head and neck cancer: 5 -year survival data from a phase 3 randomised trial, and relation between cetuximab-induced rash and survival. Lancet Oncol 2010;11:21-28.

46. Burtness B, Goldwasser MA, Flood W, et al. Phase III randomized trial of cisplatin plus placebo compared with cisplatin plus cetuximab in metastatic/recurrent head and neck cancer: an Eastern Cooperative Oncology Group study. J Clin Oncol 2005;23:86468654.

47. Vermorken JB, Mesia R, Rivera F, et al. Platinum-based chemotherapy plus cetuximab in head and neck cancer. $\mathrm{N}$ Engl J Med 2008;359:1116-1127.

48. Chen C, Kane M, Song J, et al. Phase I trial of gefitinib in combination with radiation or chemoradiation for patients with locally advanced squamous cell head and neck cancer. J Clin Oncol 2007;25:4880-4886.

49. Hainsworth JD, Spigel DR, Burris HA III, et al. Neoadjuvant chemotherapy/gefitinib followed by concurrent chemotherapy/ radiation therapy/gefitinib for patients with locally advanced squamous carcinoma of the head and neck. Cancer 2009;115:21382146.

50. Van Waes C, Allen CT, Citrin D, et al. Molecular and clinical responses in a pilot study of gefitinib with paclitaxel and radiation in locally advanced head-and-neck cancer. Int J Radiat Oncol Biol
Phys 2010;77:447-454.

51. ClinicalTrials.gov Web site. Radiation therapy and cisplatin or panitumumab in treating patients with locally advanced stage III or stage IV head and neck cancer. Available at: http://clinicaltrials. gov/ct2/show/NCT00820248?term=NCT00820248\& rank $=1$. Accessed April 4, 2011.

52. ClinicalTrials.gov Web site. Radiation therapy and cisplatin with or without cetuximab in treating patients with stage III or stage IV head and neck cancer. Available at: http://clinicaltrials.gov/ct2/ show/NCT00265941. Accessed April 4, 2011.

53. Catimel G, Verweij J, Mattijssen V, et al. Docetaxel (Taxotere): an active drug for the treatment of patients with advanced squamous cell carcinoma of the head and neck. EORTC Early Clinical Trials Group. Ann Oncol 1994;5:533-537.

54. Citrin D, Mansueti J, Likhacheva A, et al. Long-term outcomes and toxicity of concurrent paclitaxel and radiotherapy for locally advanced head-and-neck cancer. Int J Radiat Oncol Biol Phys 2009;74:1040-1046.

55. Dreyfuss AI, Clark JR, Norris CM, et al. Docetaxel: an active drug for squamous cell carcinoma of the head and neck. J Clin Oncol 1996;14:1672-1678.

56. Forastiere AA, Shank D, Neuberg D, et al. Final report of a phase II evaluation of paclitaxel in patients with advanced squamous cell carcinoma of the head and neck: an Eastern Cooperative Oncology Group trial (PA390). Cancer 1998;82:2270-2274.

57. Geng CX, Zeng ZC, Wang JY, et al. Docetaxel shows radiosensitization in human hepatocellular carcinoma cells. World J Gastroenterol 2005;11:2990-2993.

58. Cmelak AJ, Murphy BA, Burkey B, et al. Taxane-based chemoirradiation for organ preservation with locally advanced head and neck cancer: results of a phase II multi-institutional trial. Head Neck 2007;29:315-324.

59. Jain RK, Kirar P, Gupta G, et al. A comparative study of low dose weekly paclitaxel versus cisplatin with concurrent radiation in the treatment of locally advanced head and neck cancers. Indian J Cancer 2009;46:50-53.

60. Karasawa K, Shinoda H, Katsui $K$, et al. Radiotherapy with concurrent docetaxel and carboplatin for head and neck cancer. Anticancer Res 2002;22:3785-3788.

61. Sunwoo JB, Herscher LL, Kroog GS, et al. Concurrent paclitaxel and radiation in the treatment of locally advanced head and neck cancer. J Clin Oncol 2001;19:800-811.

62. Tishler RB, Busse PM, Norris CM Jr, et al. An initial experience using concurrent paclitaxel and radiation in the treatment of head and neck malignancies. Int J Radiat Oncol Biol Phys 1999;43:10011008.

63. Tishler RB, Norris CM Jr, Colevas AD, et al. A phase I/II trial of concurrent docetaxel and radiation after induction chemotherapy in patients with poor prognosis squamous cell carcinoma of the head and neck. Cancer 2002;95:1472-1481.

64. Kies MS, Harris J, Rotman M, et al. Phase II randomized trial of postoperative chemoradiation plus cetuximab for high-risk squamous cell carcinoma of the head and neck (RTOG 0234) [abstract]. Int J Radiat Oncol Biol Phys 2009;75(Suppl):S14-15. Abstract 29.

65. Barkley HT Jr, Fletcher GH, Jesse RH, Lindberg RD. Management of cervical lymph node metastases in squamous cell carcinoma of the tonsillar fossa, base of tongue, supraglottic larynx, and hypopharynx. Am J Surg 1972;124:462-467.

66. Argiris A, Stenson KM, Brockstein BE, et al. Neck dissection in the combined-modality therapy of patients with locoregionally 
Brizel et al.

advanced head and neck cancer. Head Neck 2004;26:447-455.

67. Brizel DM, Prosnitz RG, Hunter S, et al. Necessity for adjuvant neck dissection in setting of concurrent chemoradiation for advanced head-and-neck cancer. Int J Radiat Oncol Biol Phys 2004;58:1418-1423.

68. Yao M, Luo P, Hoffman HT, et al. Pathology and FDG PET correlation of residual lymph nodes in head and neck cancer after radiation treatment. Am J Clin Oncol 2007;30:264-270.

69. Robbins KT, Ferlito A, Suarez C, et al. Is there a role for selective neck dissection after chemoradiation for head and neck cancer? J Am Coll Surg 2004;199:913-916.

70. Stenson KM, Haraf DJ, Pelzer H, et al. The role of cervical lymphadenectomy afteraggressive concomitant chemoradiotherapy: the feasibility of selective neck dissection. Arch Otolaryngol Head Neck Surg 2000;126:950-956.

71. Stenson KM, Huo D, Blair E, et al. Planned post-chemoradiation neck dissection: significance of radiation dose. Laryngoscope 2006;116:33-36.

72. Tan A, Adelstein DJ, Rybicki LA, et al. Ability of positron emission tomography to detect residual neck node disease in patients with head and neck squamous cell carcinoma after definitive chemoradiotherapy. Arch Otolaryngol Head Neck Surg 2007;133:435-440.

73. Ong SC, Schoder H, Lee NY, et al. Clinical utility of 18 F-FDG
PET/CT in assessing the neck after concurrent chemoradiotherapy for locoregional advanced head and neck cancer. J Nucl Med 2008;49:532-540.

74. Nayak JV, Walvekar RR, Andrade RS, et al. Deferring planned neck dissection following chemoradiation for stage IV head and neck cancer: the utility of PET-CT. Laryngoscope 2007;117:21292134.

75. Porceddu SV, Pryor DI, Burmeister E, et al. Results of a prospective study of positron emission tomography-directed management of residual nodal abnormalities in node-positive head and neck cancer after definitive radiotherapy with or without systemic therapy. Head Neck, in press.

76. Hehr T, Classen J, Schreck U, et al. Selective lymph node dissection following hyperfractionated accelerated radio-(chemo-) therapy for advanced head and neck cancer. Strahlenther Onkol 2002;178:363-368.

77. Robbins KT, Clayman G, Levine PA, et al. Neck dissection classification update: revisions proposed by the American Head and Neck Society and the American Academy of OtolaryngologyHead and Neck Surgery. Arch Otolaryngol Head Neck Surg 2002;128:751-758.

78. Clayman GL, Johnson CJ II, Morrison W, et al. The role of neck dissection after chemoradiotherapy for oropharyngeal cancer with advanced nodal disease. Arch Otolaryngol Head Neck Surg 2001;127:135-139. 